angiography, PCI, and left ventriculography about 6 to $12 \mathrm{~h}$ after onset of AMI symptoms and were divided into two groups: the noreflow group ( 0 to 1 grade) and reflow group (2 to 3 grade) identified by MBG. Equilibrium radionuclide angiography was performed 1 week after PCI to gain the parameters of left ventricular regional and global systolic function and systolic synchrony. All patients were reinvestigated at 6 months, and major adverse cardiac events were recorded during the 6 months after PCI.

Results At 6 months after AMI-PCI, the values of left ventricular end-systolic volume index, left ventricular end-diastolic volume index, wall motion score, and left ventricular end-diastolic volume pressure in the no-reflow group were significantly increased, whereas left ventricular ejection fraction, peak ejection rate, and peak filling rate of radionuclide angiography parameters were significantly decreased $(p<0.05)$, respectively, compared with those in the reflow group. LrEF2-LrEF8 in the no-reflow group at 6 months after AMIPCI were reduced $(p<0.05)$ more than those in the reflow group, respectively. There was no improvement on the phase analysis in the no-reflow group at 6 months after AMI-PCI, and the values of PS FWHM and PSD were increased compared with those in the reflow group ( $p<0.05$; respectively). Within the 6-month follow-up period, the incidence of major adverse cardiac events in the no-reflow group was significantly higher than in the reflow group.

Conculsion The infarct-related zone myocardium post-AMI still has no reperfusion status and directly causes the reduction of the regional and global left ventricular systolic performance with an increase of systolic asynchrony. It then progressively decreases the efficiency of ventricular blood ejection as well as causes ventricular remodelling with adverse long-term outcome in patients with AMI.

\section{e0644 ESTABLISHMENT OF CONTRAST INDUCED NEPHROPATHY MODEL IN RATS}

doi:10.1136/hrt.2010.208967.644

Fu Xianghua, Jia Xinwei, Wang Yanbo, Wang Xuechao, Zhang Jing, Fan Weize, Gu Xinshun, Jiang Yunfa. The 2nd Hospital of Hebei Medical University, Shijiazhuang, Hebei, China

Objectives The purpose of this study was to establish an rat model of CIN and to evaluate its efficacy.

Methods Totally $24 \mathrm{SD}$ rats were randomly allocated into experimental group (group $\mathrm{A}, \mathrm{n}=12$ ) and control group (group $\mathrm{B}, \mathrm{n}=12$ ). After dehydration for 3 days, rats in group A were given intravenous MDDS, while rats in group B were given intravenous normal saline. Then, all rats got normal water-drinking to the end of study. Renal ultrasonic examination was performed to observe the morphologic changes, diameters of renal artery and blood flow in renal artery. Blood samples were taken to measure the level of serum creatinine. The tissue of kidney were incised for microscope and electron microscope study.

Results The dimensions of the two groups before and after dehydration were not different. It gradually enlarged after CM injection. These changes were the most obvious at 6 and $12 \mathrm{~h}$, which did not recover at $24 \mathrm{~h}$. The PSV, EDV, S/D and VTI were lowerest at $6 \mathrm{~h}$ and then recover to normal level at $24 \mathrm{~h}$. RI was increased after CM injection, the lowest occurred at $6 \mathrm{~h}$, and recovered to normal level at $24 \mathrm{~h}$. Serum creatinine was significantly elevated after dehydration, the highest level occurred at $12 \mathrm{~h}$ and then began to recover at $24 \mathrm{~h}$. Microscope examination to renal sample at $12 \mathrm{~h}$ found patch disappearance of tubular structure, widely congestion at medullar area. No pathological glomerular changes were found under microscope. Electron microscope examination found desquamation, sparseness of microvillous of tubular endothelium, membrane confusion, disappearance, swelling, fragmentation of the MIT, with obstructed tubular lumen and basal membrane swelling.
Conclusion Combined with dehydration, intravenous injection of contrast lead to obvious acute kidney injury, with the changes of kidney tissue pathology, haemodynamics and kidney functions which are similar to the characteristics of CIN in human beings.

\section{E0645 PROTECTIVE EFFECTS OF SIMVASTATIN COMBINED WITH ANISODAMINE ON MYOCARDIAL PERFUSION IN SWINE NO REFLOW MODEL}

doi:10.1136/hrt.2010.208967.645

Fu Xianghua, Jia Xinwei, Wang Yanbo, Wang Xuechao, Zhang Jing, Fan Weize, Hao Guozhen. The 2nd Hospital of Hebei Medical University, Shijiazhuang, Hebei, China

Objectives To evaluated the preventive effect of simvastatin combined with anisodamine on myocardial perfusion in no reflow, and to probe the possible mechanism.

Method Totally 16 minipig of $30-40 \mathrm{Kg}$ were randomly divided into anisodamine groups $(A, n=8)$ and anisodamine plus simvastatin group $(A+S, n=8)$. Pigs in Group $A+S$ were pretreated with oral simvastatin for 7 days, while pigs in A groups were given placebo. Seven days later, CAG was performed, and the dopper wire was used to record blood velocity. The pressure of aorta $(\mathrm{Pa})$ was monitored. PMBS was injected to establish no reflow model. Anisodamine was injected into the LAD 2 min before PBMS was injected. The TIMI blood flow, TMPG and CTFC were recorded to evaluate the myocardial perfusion. The sample of myocardium in ischaemic zone and normal zone were measured. Blood sample was taken before and after the expeiment to measure the level of CK-MB, cTnI and hs-CRP. The percent of necrostic myocardium was calculated by myocardium stain method. Results The TIMI blood flow and TFCs were better in Group $\mathrm{A}+\mathrm{S}$ $(p<0.05)$. The Pa was increased in both groups after PMBS injection at the early stage $(p<0.01)$, and then it begun to decrease in Group A $(p<0.05)$, while it remained its high level in Group $A+S(p=0.042)$. The bAPV was increased in both groups, which was more obvious in the Group A after PMBS injection. After the injection of PMBS, the hAPV was sinificantly decreased in both groups $(p<0.01)$, but it was still higher in group $A+S(p=0.000)$. The CFR was continously deceased after the PMBS injection $(p<0.05)$, but it was higher in Group $A+S(p=0.025)$. The h-MR was further increased $(p=0.024)$, with no difference between two groups after the PMBS injection. The level of serum cholesterol was similar between the two groups $(p=0.063)$. CK$\mathrm{MB}$, TnI, hs-CRP and MDA were increased after the experiment, with the higher levels in Group A. NO was also increased $(p=0.000)$, with the higher level in Group $A+S(p=0.006)$. SOD was decreased $(p=0.000)$ in both groups, with lower level in Group $A(p=0.000)$. The infarcted size in group A was larger than that in $A+S$ group $(p<0.05)$. Conclusion Simvastatin combined with anisodamine can significantly improve myocardial blood perfusion and porotect the myocardium against ischaemic injury during PCI. The possible mechanism involves improving of coronary haemodynamics, antiinflammation and antioxidation.

\section{e0646 PROTECTIVE EFFECT OF INTENSIVE STATIN PRETREATMENT ON RENAL FUNCTION IN PATIENTS WITH ACUTE CORONARY SYNDROME UNDERGOING PERCUTANEOUS INTERVENTION}

doi:10.1136/hrt.2010.208967.646

Fu Xianghua, Jia Xinwei, Wang Yanbo, Wang Xuechao, Zhang Jing, Fan Weize, Hao Guozhen, Jiang Yunfa. The 2nd Hospital of Hebei Medical University, Shijiazhuang, Hebei, China

Objectives To evaluate the protective effects of higher dose statin on renal function and the incidence of CIN. 